\title{
Hyperspectral system for Imaging of skin chromophores and blood oxygenation
}

E. Zherebtsov, A. Popov, A. Doronin, I. Meglinski, A. Bykov

E. Zherebtsov, A. Popov, A. Doronin, I. Meglinski, A. Bykov, "Hyperspectral system for Imaging of skin chromophores and blood oxygenation," Proc. SPIE 10412, Diffuse Optical Spectroscopy and Imaging VI, 104120G (28 July 2017); doi: $10.1117 / 12.2280779$

SPIE. Event: European Conferences on Biomedical Optics, 2017, Munich, Germany 


\title{
Hyperspectral System for Imaging of Skin Chromophores and Blood Oxygenation
}

\author{
E. Zherebtsov ${ }^{* 1}$, A. Popov ${ }^{2}$, A. Doronin ${ }^{3}$, I. Meglinski ${ }^{2}$, A. Bykov ${ }^{2}$ \\ ${ }^{1}$ Aston University, Optoelectronics and Biomedical Photonics Group, Aston Institute of Photonic Technologies, \\ Aston Triangle, Birmingham B47ET, UK \\ ${ }^{2}$ University of Oulu, Opto-Electronics and Measurement Techniques Research Unit, \\ Faculty of Information Technology and Electrical Engineering, P.O. Box 4500, 90014 Oulu, Finland \\ ${ }^{3}$ Yale University, Department of Computer Science, Computer Graphics Group, New Haven, CT, 06520, USA \\ *Correspondence: e.zherebtsov@aston.ac.uk
}

\begin{abstract}
We developed a compact, fast, hand-held hyperspectral imaging system for 2D neural network-based visualization of skin chromophores and blood oxygenation. Here, we present results of the system tests on healthy volunteers.

OCIS codes: (170.0110) Imaging systems; (170.6510) Spectroscopy, tissue diagnostic
\end{abstract}

\section{Introduction}

Methods of in vivo skin chromophore assessment and blood oxygen measurements have a long history. ${ }^{1}$ Conventional techniques utilize measurements at several wavelengths in the visible and NIR ranges (usually 2-4 wavelengths) or color sensors rather than real spectral measurements. ${ }^{2,3}$ However, to our knowledge, no portable, compact, lightweight, hand-held and robust hyperspectral devices capable of skin chromophore content analysis (2D mapping), skin perfusion and monitoring of blood oxygenation at the same time have been presented up to date.

Spectral images of skin and other tissues contain information about the spatial distribution and concentrations of biological chromophores, such as hemoglobin (oxy-/deoxy), melanin, bilirubin, and carotene. ${ }^{4,5}$ The combination of a fast and compact hyperspectral camera with a broadband light illumination and neural network-based processing allows for multiparameter estimation of tissue properties. Potential applications of the proposed technique are of high social impact and include monitoring and diagnostics of diabetic ulcer formation, ${ }^{6}$ rheumatic complications, ${ }^{7,8}$ diagnoses of melanoma and other malignancies, ${ }^{9}$ quantitative skin screening, ${ }^{10}$ wound healing control, ${ }^{11}$ assistance and support of laser surgical and treatment procedures ${ }^{12}$ and others.

Thus, the aim of the present study is the development of a new approach to hyperspectral imaging for quantitative diagnostics and characterization of skin including 2D mapping of skin chromophore (hemoglobin, melanin) distribution, kinetics of blood oxygenation maps and evaluation of skin perfusion.

\section{Methods and Materials}

In this study, two-dimensional spatially resolved diffuse reflectance of human skin was acquired with a compact hand-held hyperspectral snapshot camera, thus avoiding spatial scanning. The camera implements a micro FabryPerot interferometer and has the spectral resolution of 6-10 nm (FWHM) in the spectral range of 500-900 nm. A laboratory-build broadband illumination unit is based on a fiber-optic ring illuminator providing uniform distribution of light intensity in the camera focal plane and utilizes a 50W halogen lamp as a source. Photo and the schematics of the developed device are presented in Fig.1. Diffuse reflectance of the skin was measured with the spectral step of 2 $\mathrm{nm}$ from the area of $5 \times 5 \mathrm{~cm}(1010 \times 1010$ pixels).

To solve the inverse problem of estimation of both skin chromophore content and the values of blood oxygenation, an approach based on the neural network has been chosen. A training set of spectra for the neural network was modelled by the Monte Carlo method. A CUDA-based Monte-Carlo distributed computing platform was used for routine simulation of diffuse reflectance spectra of skin, as well as their variations associated with possible physiological changes in the human skin. ${ }^{13}$ The differences in the spatial distribution of blood, melanin, oxygen saturation of blood, hematocrit, water content within the skin, as well as the numerical aperture and the angle of the detector positioning on the skin surface were taken into account. To model the diffuse reflectance spectra, a sevenlayer model of skin was used. ${ }^{14}$ The main parameters of the model are presented in Table 1. In the table, parameters $K_{b}, S, K_{m e l}, K_{e p i}$ were varied to model all the possible combinations of the parameters. For this purpose, the coefficient of the blood volume fraction $K_{b}$ was varied in a range of $0-3$ with a step of 0.1 . The blood oxygen

Diffuse Optical Spectroscopy and Imaging VI, edited by Hamid Dehghani, Heidrun Wabnitz, Proc. of SPIE-OSA Vol. 10412, 104120G · C) 2017 OSA-SPIE

CCC code: $1605-7422 / 17 / \$ 18 \cdot$ doi: $10.1117 / 12.2280779$ 
saturation $S$ was varied in a range of $20-100 \%$ with a step of $1 \%$. The coefficient of melanin fraction $K_{m e l}$ was varied in a range of 0-9 with a step of $1 .{ }^{15}$ And the coefficient of epidermis thickness was varied in a range of 1-3 with a step of 1 . The total training set had 45198 spectra in the range of 505-800 nm with a step of $5 \mathrm{~nm}$.
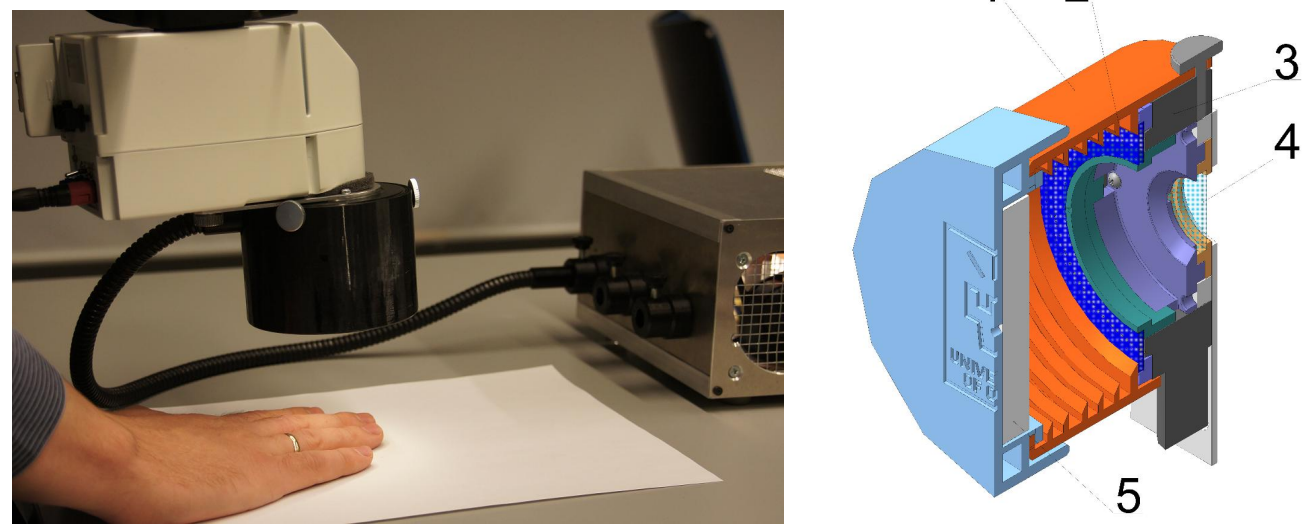

Fig.1. Photo and schematic design of the developed prototype of hyperspectral imaging system. 1 - lens hood (spacer); 2 - ring-polarizer for illumination; 3 - fiber illumination unit; 4 - camera polarizer; 5 - build in diffuse reflectance standard.

In the considered model, the absorption coefficient of each layer takes into account the concentration of blood $C_{\text {blood }}$ in various vascular beds, oxygen saturation $S$, water content $C_{H 2 O}$, melanin fraction $C_{m e l}$ and are defined as: ${ }^{14}$

$$
\begin{gathered}
\mu_{a}^{\text {strat.corneum }}(\lambda)=\left(1-C_{\mathrm{H}_{2} \mathrm{O}}\right) \mu_{a}^{\text {baseline }}(\lambda)+C_{\mathrm{H}_{2} \mathrm{O}} \mu_{a}^{\text {water }}(\lambda), \\
\mu_{a}^{\text {epidermis }}(\lambda)=\left(1-C_{\mathrm{H}_{2} \mathrm{O}}\right)\left[C_{\text {mel }} \mu_{a}^{\text {mel }}(\lambda)+\left(1-C_{\text {mel }}\right) \mu_{a}^{\text {baseline }}(\lambda)\right]+C_{\mathrm{H}_{2} \mathrm{O}} \mu_{a}^{\text {water }}(\lambda), \\
\mu_{a}^{\text {laver }}(\lambda)=\left(1-C_{\mathrm{H}_{2} \mathrm{O}} \mathrm{O}\right)\left[C_{\text {blood }} H t\left(S \mu_{a}^{\text {oxy }}(\lambda)+(1-S) \mu_{a}^{\text {deoxy }}(\lambda)\right)+\left(1-C_{\text {blood }} H t\right) \mu_{a}^{\text {baseline }}(\lambda)\right]+C_{\mathrm{H}_{2} \mathrm{O}} \mu_{a}^{\text {water }}(\lambda) .
\end{gathered}
$$

Here, $\mu_{a}^{m e l}$ is the absorption coefficient of melanin, $\mu_{a}^{\text {baseline }}$ is the absorption coefficient of water-free tissues, $\mu_{a}^{\text {water }}$ is the absorption coefficient of water, $\mu_{a}^{\text {deoxy }}$ and $\mu_{a}^{o x y}$ are the absorption coefficients of reduced and oxygenated haemoglobin respectively, $H t=0.45$ is hematocrit index. The scattering coefficients of the layers are approximated based on the combination of Mie and Rayleigh scattering: ${ }^{16,17}$

$$
\mu_{s}^{\text {layer }}=3.3\left(2.2 \cdot 10^{11} \cdot \lambda^{-4}+11.74 \cdot \lambda^{-0.22}\right) .
$$

Table 1. Parameters used for assessment of the absorption coefficients of the skin layers

\begin{tabular}{|l|c|c|c|c|c|}
\hline \multicolumn{1}{|c|}{ Layer } & Thickness, $\mu \mathrm{m}$ & $\begin{array}{c}\text { Blood volume } \\
\text { fraction } C_{\text {blood }} \%\end{array}$ & $\begin{array}{c}\text { Saturation, } \\
\%\end{array}$ & $\begin{array}{c}\text { Water } C_{H 2 O}, \\
\%\end{array}$ & $\begin{array}{c}\text { Melanin } \\
C_{\text {mel }}, \%\end{array}$ \\
\hline Stratum corneum & $20 K_{e p i}$ & 0 & 0 & 5 & 0 \\
\hline Living epidermis & $60 K_{e p i}$ & 0 & 0 & 20 & $K_{\text {mel }}$ \\
\hline Papillary dermis & 250 & $5 K_{b}$ & $S$ & 30 & 0 \\
\hline $\begin{array}{l}\text { Upper blood net } \\
\text { dermis }\end{array}$ & 330 & $20 K_{b}$ & $S$ & 40 & 0 \\
\hline Reticular dermis & 1830 & $4 K_{b}$ & $S$ & 50 & 0 \\
\hline $\begin{array}{l}\text { Deep blood net } \\
\text { dermis }\end{array}$ & 1910 & $10 K_{b}$ & $S$ & 50 & 0 \\
\hline Subcutaneous fat & 8000 & $7 K_{b}$ & $S$ & 60 & 0 \\
\hline
\end{tabular}

The Matlab neural network fitting tool was chosen to build and train 4 neural networks for retrieval of each of the parameters $\left(K_{b}, S, K_{m e l}, K_{e p i}\right)$. Analysis of the obtained training set of spectra allowed selecting the most prospective wavelength ranges for the fitting. In the proposed approach, the blood volume fraction coefficient $K_{b}$ is retrieved in the spectral range of 510-630 nm, the blood oxygen saturation $S$ is retrieved in the spectral range of $660-800 \mathrm{~nm}$, the coefficient of melanin fraction $K_{m e l}$ is retrieved in the range of $660-720 \mathrm{~nm}$, the epidermis thickness coefficient $K_{e p i}$ is retrieved in the spectral range of $680-800 \mathrm{~nm} .{ }^{18}$ Before the neural network training procedure, the vectors of the 
corresponding spectral subranges for every assessed parameter were normalized. Preprocessing of the measurement data consists of the spatial averaging of the input hyperspectral cube in a $5 \times 5$ pixel window. After the averaging, the corresponding data vectors used for assessing the parameters $\left(K_{b}, S, K_{m e l}, K_{e p i}\right)$ are normalized.

The developed hyperspectral imaging system was used to perform trial measurements and the occlusion tests with healthy volunteers. Finally, two-dimensional maps of the blood volume fraction, the blood oxygen saturation and the melanin content of the skin were obtained.

\section{Result and Discussion}

Typical images of the blood volume fraction distribution during occlusion of the middle finger are presented in Fig. 2. After the assessment of $K_{b}$ parameter, an average blood volume level was calculated for the analyzed skin layers. Typical images of blood oxygen saturation distribution during the same experiment are presented in Fig. 3. Comparison of the obtained melanin fraction distributions in the Indian and Caucasian skin are shown in Fig. 4.

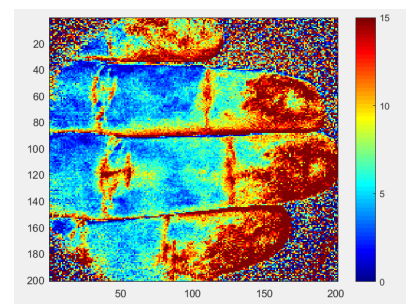

a)

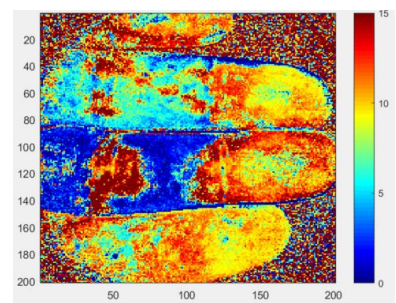

b)

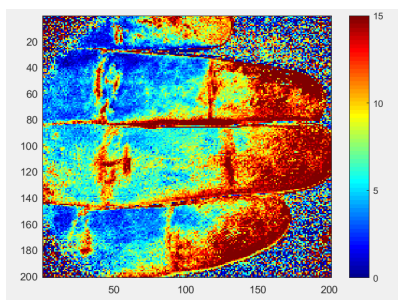

c)

Fig. 2. Obtained images of the blood volume fraction distribution before (a), during (b) and after (c) middle finger occlusion.

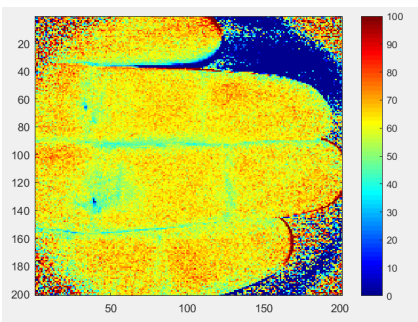

a)

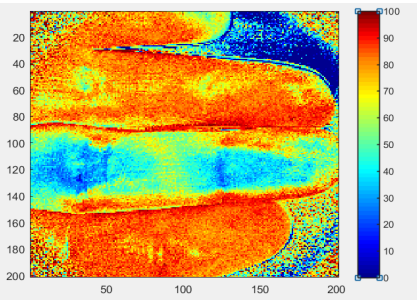

b)

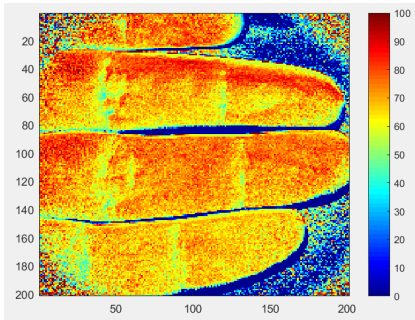

c)

Fig. 3. Obtained images of blood oxygen saturation before (a), during (b) and after (c) middle finger occlusion.

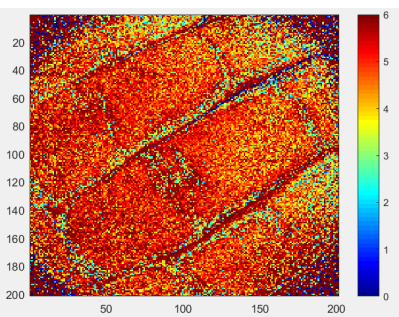

a)

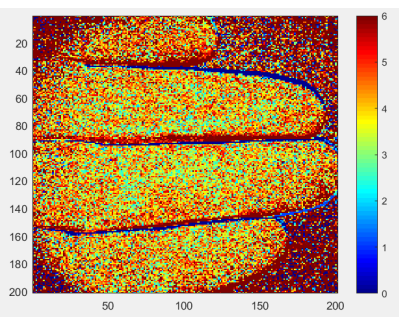

b)

Fig. 4. Obtained images of melanin fraction distribution in Indian (a) and Caucasian (b) skin.

Thus, the developed hyperspectral system has shown the ability to sense the alterations of blood and melanin content in skin as well as blood oxygen saturation. The implementation of the neural network-based processing allows for fast recovery of the considered skin parameters. The mean processing time of one hyperspectral measurement including calculation of 2D distributions of the above-mentioned skin parameters on an Intel Core i7 computer with $16 \mathrm{~Gb}$ RAM requires approximately $40 \mathrm{~s}$. 


\section{Conclusion}

A compact, fast, hand-held hyperspectral imaging system for 2D neural network-based visualisation of blood volume fraction, saturation and melanin fraction distribution in the skin was developed. The system was used to perform the trial measurements and occlusion tests with healthy volunteers. As a result, the two-dimensional maps of blood volume fraction, blood oxygen saturation and melanin content of skin were obtained. The developed technique has potential applications in monitoring and diagnostics of diabetic ulcer formation and other relevant skin diseases along with cosmetological defects.

\section{Acknowledgments}

Authors acknowledge the support of the Academy of Finland (grants: 296408 and 290596). Evgeny Zherebtsov also acknowledges the support provided by H2020 MCSA funded project, grant number 703145 .

\section{References}

[1] V.V. Tuchin, [Tissue Optics: Light Scattering Methods and Instruments for Medical Diagnosis], Society of Photo-Optical Instrumentation Engineers (SPIE), 841 (2015).

[2] L.L. Randeberg, E.L.P. Larsen, L.O. Svaasand, "Characterization of vascular structures and skin bruises using hyperspectral imaging, image analysis and diffusion theory," J. Biophotonics 3(1-2), 53-56 (2009).

[3] J. Spigulis, I. Oshina, A. Berzina, A. Bykov, "Smartphone snapshot mapping of skin chromophores under triplewavelength laser illumination", J. Biomed. Opt., 22(9), 091508 (2017).

[4] D. Kapsokalyvas, N. Bruscino, D. Alfieri et al., "Spectral morphological analysis of skin lesions with a polarization multispectral dermascope," Opt. Express 21(4), 4826-4840 (2013).

[5] J. O'Doherty, P. McNamara, N.T. Clancy, "Comparison of instruments for investigation of microcirculatory blood flow and red blood cell concentration," J. Biomed. Opt. 14(3), 034025 (2009).

[6] V.V. Dremin et al., "The blood perfusion and NADH/FAD content combined analysis in patients with diabetes foot," Proc. SPIE 9698, 969810 (2016).

[7] E.A. Zherebtsov et al., "Combined use of laser Doppler flowmetry and skin thermometry for functional diagnostics of intradermal finger vessels," J. Biomed. Opt. 22(4), 40502 (2017).

[8] A.I. Zherebtsova et al., "Study of the functional state of peripheral vessels in fingers of rheumatological patients by means of laser Doppler flowmetry and cutaneous thermometry measurements," Proc. SPIE 9917, 99170M (2016).

[9] A. Bykov et al., "Polarization sensitive optical biopsy with diffusely reflected polarized light," OSA Technical Digest JTu3A, JTu3A.35 (2016).

[10] M.V. Volkov et al., "Evaluation of blood microcirculation parameters by combined use of laser Doppler flowmetry and videocapillaroscopy methods," Proc. SPIE 10336, 1033607 (2017).

[11] A.V. Bykov, A.V. Priezzhev, and R. Myllyla, "Spatial resolved diffuse reflection as a tool for determination of size and embedding depth of blood vessels," Diffuse Optical Imaging of Tissue 6629, 61 (2007).

[12] A.V. Dunaev, "Method and installation used for testing of the absorbed dose of radiation during low-level laser therapy," Proc. SPIE 6440, 64400T (2007).

[13] A. Doronin, I. Meglinski, "Online object oriented Monte Carlo computational tool for the needs of biomedical optics," Biomed. Opt. Express, 2(9), 2461-2469 (2011).

[14] G.I. Petrov et al., "Human tissue color as viewed in high dynamic range optical spectral transmission measurements," Biomed. Opt. Express, 3(9), 2154-2161 (2012).

[15] V.V. Dremin and A. V Dunaev, "How the melanin concentration in the skin affects the fluorescencespectroscopy signal formation," J. Opt. Technol. 83(1), 43 (2016).

[16] M.S. Wróbel et al., "Measurements of fundamental properties of human skin phantoms", J. Biomed. Opt. 20(4), 045004 (2015).

[17] A.V. Bykov et al., "Multilayer tissue phantoms with embedded capillary system for OCT and DOCT imaging", Proc. SPIE, 8091, 80911R (2011).

[18] A.V Dunaev et al., "Substantiation of medical and technical requirements for noninvasive spectrophotometric diagnostic devices.," J. Biomed. Opt. 18(10), 107009 (2013). 